\title{
Measuring Aesthetics for Information Visualization
}

\author{
Daniel Filonik, Dominikus Baur \\ Media Informatics Group, University of Munich \\ daniel.filonik@stud.ifi.lmu.de, dominikus.baur@ifi.lmu.de
}

\begin{abstract}
Aesthetics is an unsolved problem of information visualization, because there is no satisfactory understanding of what constitutes aesthetic effect. This survey paper gives an overview of approaches to model aesthetics, starting with Birkhoff's aesthetic measure and continuing to recent ones based on mathematical and information theoretical concepts. Common concepts in the different models are highlighted, such as the effects of order and complexity. Further, practical techniques for generating aesthetic visualizations are shown together with examples of recent work in this field. Finally, the paper discusses some of the key issues regarding aesthetics and the human factor in the visualization process. Empirical studies have shown a correlation between perceived aesthetics and usability, meaning that a better understanding of aesthetics could improve the usability of visualizations.
\end{abstract}

Keywords-Aesthetics, Visualization, Measures, Usability

\section{Introduction}

Since its formal definition in the late 1980s the field of interactive computer-aided visualization has experienced rapid development. However, despite the notable progress, there are still numerous open questions. Some of the most pressing ones are listed in Chaomei Chen's article "Top 10 Unsolved Information Visualization Problems" [4].

In the context of this paper, the mentioning of aesthetics as one of the unsolved problems is certainly worth highlighting. As Chen points out "there is a lack of holistic empirical studies to characterize what visual properties make users think a graph is pretty or visually appealing" [4]. Therefore, it is important to look at approaches for measuring aesthetics, which are not based on a specific kind of visualization. The connections between aesthetics and insight need to be explored in order to make visualizations that are attractive and effective. Further, when investigating the role of aesthetics it is necessary to consider its implications for methodologies aiming to evaluate visualizations.

Besides aesthetics, Chen also considers the issue of usability. Empirical studies have shown that perceived aes- thetics have a positive effect on usability [3,22]. This is a critical issue, since the user plays a central role in the visualization process. The interactive nature of visualizations poses high demands in terms of usability. Aesthetics should not be reduced to pleasing visual design, aesthetic qualities in interaction design also have to be considered when evaluating visualizations $[4,6]$.

With robust aesthetic measurements in place it might even be possible to solve the lack of intrinsic quality measures. Measures for aesthetics could work as benchmarks to compare different visualizations [4].

Consequently, aesthetics is not only an unsolved problem in the field of visualization, but it can be related to other unsolved problems. Further research into this topic could prove very beneficial for the visualization community. This paper aims to give an overview over relevant work and different approaches to this topic. The following chapter takes a closer look at the central terms. Afterwards, the focus lies on different theoretical approaches to measure aesthetics. Existing techniques for generating aesthetic images are examined and examples are presented. The final chapter is concerned with the effect of aesthetic visualizations on the user.

\section{Defining Aesthetics}

In its modern use "aesthetics" usually refers to the philosophical study of art and beauty. The word is originally derived from the Greek "aisthesis". In its original use it covered the meaning of the English terms sensation, perception, appearance, mind and knowledge [12]. In search for a good definition for aesthetics, Cawthon and Moere turn to its antonym.

"An anesthetic is used to dull or deaden, causing sleepiness and numbness. In contrast, aesthetic is seen as something that enlivens or invigorates both body and mind, awakening the senses.” [2]

There are various academical approaches to aesthetics. Analytical aesthetics, which is represented in this paper through aesthetic measures, is based on the logical and 
mathematical nature of aesthetics. In art, psychology or industrial product design the approaches are very different [3]. The user has a subjective view of aesthetics as a result of his personal, social and cultural background. Therefore, there is no single theory of aesthetics.

In software development, aesthetics is most frequently considered in regard to interface design. However, it is often seen as an added bonus that requires additional programming time and resources. There may even be negative connotations, as an attractive interface is suspected to cover up deficiencies in functionality [2]. Such allegations loose ground as there are empirically measurable benefits of aesthetics $[3,22]$. In the wake of such findings it is apparent that the subject deserves greater attention then it has previously received [15].

\section{Aesthetic Measures}

While it may be true that "no one knows how to measure aesthetic value" [15], several analytical approaches have been developed to quantify certain aspects of aesthetic appearance. This section first examines the original work "Aesthetic Measure" [1] by George D. Birkhoff, which suggested a formalization of aesthetics. Since then, different approaches have been made building up on the fundamental insights of Birkhoff's work.

\subsection{Birkhoff's Aesthetic Measure}

The goal of Birkhoff's measure was to determine the aesthetic effect of objects such as polygons, tiles and vases. Birkhoff applied his model only to a limited range of objects, because he came to the conclusion that objects of different classes could not be compared. He also realized that aesthetic effect was dependent on the observer and proposed a restricted group of observers $[18,21]$.

The early mathematical model of beauty proposed by Birkhoff was based on three phases in the perception of aesthetics. The first phase is the preliminary effort of focusing one's attention on the object. This phase is proportional to the object's complexity $(C)$. The reward of this effort is a feeling of value, which is called the aesthetic measure $(M)$. However, this aesthetic measure is influenced by the degree of harmony, symmetry or order $(O)$. The verification of order in the object seems necessary for the aesthetic effect [18]. From these observations, Birkhoff deduced the following relationship.

$$
M=\frac{O}{C}
$$

This formula can be interpreted quite intuitively. A rising degree of complexity and disorder creates an unpleasant reaction from the viewer. In the model this is represented by a lower value of the aesthetic measure. Inversely, a higher amount of order results in a more satisfying experience, signaled by a higher aesthetic measure. Later studies with groups of observers suggested that the perceived aesthetic value "is curvilinearly related to $M$, peaking when $M$ is moderate" [21].

The intuitive interpretation and frequent reoccurrence in other works shows that Birkhoff's measure is very appealing. However, trying to apply it to information visualization poses a new question: how is complexity and order measured? New approaches try to answer this question, based upon work in information theory and mathematical concepts.

\subsection{A Pattern Measure}

Klinger and Salingaros' measure [11] holds some close similarities to Birkhoff's measure. The class of objects covered by Klinger and Salingaros are rectangular square arrays of elements, also referred to as patterns. Evidently, such a measure is particularly interesting if visualizations are made up of elements placed in a grid. Moreover, it can be applied to any raster of pixels.

The cognitive process is again the underlying principle for formulating a model of aesthetics. During perception the viewer subliminally identifies coherent units and notices how often identical units appear. These factors contribute to the viewers impression of the object. Mathematically simple patterns are easily recognized as opposed to random ones.

The pattern measure combines hierarchy with information measures to estimate the complexity and order of a pattern. The result are two descriptors: $T$ represents traditional measures of information and $H$ represents symmetries. This means that $T$ can be regarded as a measure for complexity and $H$ as a measure of order. A measure for disorder can then be derived by subtracting $H$ from the maximum possible symmetry $H_{\max }$. From these descriptors Klinger and Salingaros derive the following two composite measures.

$$
\begin{gathered}
L=T H \\
C=T\left(H_{\max }-H\right)
\end{gathered}
$$

The composite measure $L$ corresponds with the degree of structure, whereas $C$ represents randomness. Klinger and Salingaros suggest that the mathematical quantities $T$ and $H$ are not perceived directly, while $L$ and $C$ are. Both composite values have to be considered as separate dimensions when estimating the aesthetic effect.

The calculation of the pattern measure in the simple case of a $2 \times 2$ array is relatively easy. The value of $T$ is the number of different elements in the array minus one. There are six possible symmetries in such a simple array - four reflectional symmetries along different axis and two 
rotational symmetries. The value for $H$ can easily be obtained by checking each of the possible symmetries.

In order to be able to apply the measure to larger arrays, Klinger and Salingaros decompose them into sub blocks. This subdivision is applied recursively until the case of the $2 \times 2$ array is reached. When moving up in the recursion the values calculated in the sub blocks are accumulated. In addition to the six symmetries of the simple $2 \times 2$ case, three translational symmetries have to be considered: relation through translation and reflection, relation through translation and rotation and similarity to another element.

\subsection{Informational Aesthetics Measures}

The work of Rigau et al. [18] devises a set of measures that build on Birkhoff's with information theoretical concepts. The area of application in the paper are digital images, which means that it could be applied to the output of visualization algorithms.

Different concepts in the creative process form the basis for the proposed model. On the one hand, the initial repertoire is a finite and discrete palette of colors available for the creation of an image. On the other hand, the selected repertoire is the range of colors that were used with a certain probability distribution. The final distribution of colors on the canvas that makes up the image.

The initial repertoire is given by the standard RGB palette, which has $N^{r g b}=256^{3}$ values. The maximum entropy can therefore be calculated as $H_{\max }=$ $\log _{2}\left(N^{r g b}\right)=24$. The selected repertoire is referred to as $X^{r g b}$. The intensity histogram represents the probability distribution of colors in the image. With this probability distribution it is possible to calculate the Shannon entropy $H\left(X^{r g b}\right)$ of the image. This information can be used to calculate the following measure.

$$
M_{B}=\frac{H_{\max }-H\left(X^{r g b}\right)}{H_{\max }}
$$

The next measure introduces the concept of Kolmogorov complexity. Essentially, Kolmogorov complexity of a string is the length of its ultimate compressed version. This value cannot be computed, but it can be estimated by using standard real world compression algorithms. The number of pixels in the image $(N)$ is required for calculating the size an uncompressed version $\left(N H_{\max }\right)$. This size is put in relation to the Kolmogorov complexity $(K)$ approximated with the use of JPEG compression.

$$
M_{K}=\frac{N H_{\max }-K}{N H_{\max }}
$$

Finally, a measure is defined based on physical entropy, that relates the images initial information content to the Kolmogorov complexity.

$$
M_{Z}=\frac{N H\left(X^{r g b}\right)-K}{N H\left(X^{r g b}\right)}
$$

These measures are used to quantify the creative process. The value of $M_{B}$ represents the selection of colors from the initial repertoire and $M_{K}$ the order in the distribution of colors. The last measure $M_{Z}$ "expresses the transition from the palette to the artistic object".

The paper goes on to devise a partitioning algorithm that aims at maximizing the extraction of information at each step. The goal is to model the process of an observer trying to decompose the image. The more steps are necessary to achieve a certain level of information, the higher the complexity of the image.

\subsection{Hereditary Combinatorial Entropy}

Combinatorial entropy is proposed by Nešetřil [14] for measuring harmony in an image. It is invariant in regard to scaling and rotation and very robust. Using digital image processing tools it can be applied to a wide range of images, some of which may have to be preprocessed for contours.

The measure is based on the image represented as a finite set of curves in two-dimensional Euclidean space. For a set of curves $D$ and an infinite straight line $L$, the number of intersections is denoted by $i(L, D)$. This leads to the definition of the combinatorial entropy as follows.

$$
H_{c}(D)=\operatorname{Ex}(i(L, D))
$$

That means that combinatorial entropy denotes the expected value of intersections related to a random selection of a line $L$. The mathematical definition of this expected value is difficult. However, $H_{c}(D)$ can be easily evaluated by a sufficiently large number randomly generated of lines. When applied to the simple case of a $n \times n$ grid, the value of $H_{c}(D)$ is $n$.

An interpretation of combinatorial entropy is that it represents the information content of an image. When comparing images of the same object or theme, a "smaller $H_{c}(D)$ indicates the relative elegance and simplicity of the output".

Introducing a hierarchical structure to the process, hereditary combinatorial entropy $(H C E)$ is defined based on $H_{c}(D)$. It "can be visualized as a matrix that has a hereditary structure, where each field has a corresponding combinatorial entropy". The goal of $H C E$ is to take features such as clustering or equidistribution of lengths and points into account, which cannot be computed easily.

Nešetřil concludes that "a harmonious or aesthetically pleasing drawing or design has a combinatorial entropy in each of its (meaningful) parts proportional to the global combinatorial entropy". 


\section{Aesthetic Visualization Approaches}

This section is concerned with the generation of aesthetic appearance based on the formalization of aesthetics and particular qualities of different algorithms. Sometimes, this is also referred to as algorithmic or generative art. Work in this area is characterized by experimentation with different creative approaches and often has an essential component of visual interaction. Several technologies for displaying advanced and highly interactive graphics in a browser have made the Internet a popular platform for presenting such work [10].

\subsection{Exact Aesthetics}

The field of exact aesthetics deals with the reconstruction of methods of design and criticism on algorithmic basis. The aesthetic measures are one important starting point for the development of such algorithms. The goal is to integrate a computer into processes of artistic creation and aesthetic evaluation.

Staudek and Machala [20] developed an exact aesthetics application named Arthur that designs abstract images based on a set of rules. The output is defined by a grammar and a repertoire of symbols, each of which can be modified by the user. The generation of the image is controlled by criteria such as symbols arrangement, coloring, contrast or appearance preferences.

A key component of the program is the implementation of Klinger and Salingaros' pattern measure for evaluating the aesthetic effect of the generated pattern. The program repeats algorithmic creation and evaluation until a certain aesthetic level is reached. The same approach could be applied to the generation of information visualizations.

The pattern shown in figure 1 is evaluated by the application as being "complex, low-ordered yet considerably attractive".

Staudek and Machala are planning to enrich the original program "with additional aesthetic functions for evaluating proportions, cohesion, homogeneity, or rhythm of patterns". Continued research and new approaches to aesthetic measures play a major role in further improving the quality images produced by exact aesthetics.

\subsection{Genetic Algorithms}

Genetic algorithms are inspired by evolutionary processes in nature. Such algorithms have a large variety of applications, ranging from simulation of living ecosystems to computer vision research. In particular, genetic algorithms have been picked up by modern artists for generating imagery or even music [10].

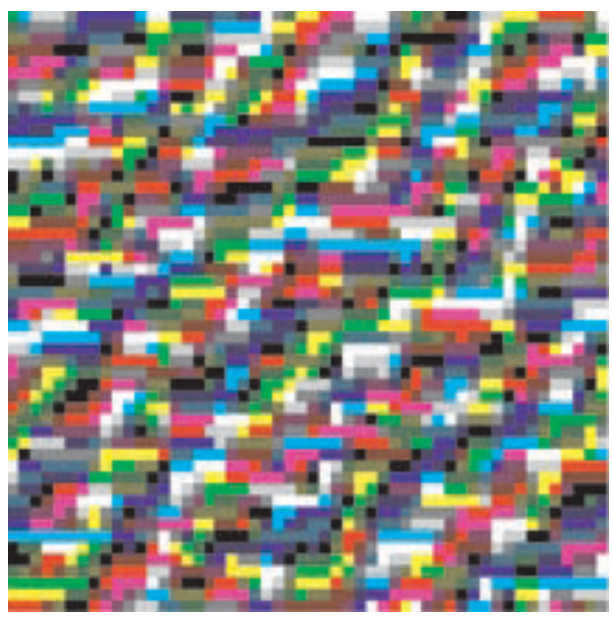

Figure 1: Pattern generated by the Arthur application [20].

In a genetic algorithm, the computer simulates the process of evolution on a group of random individuals, also referred to as chromosomes. Each chromosome represents a candidate solution to the problem at hand. The selection of chromosomes that pass their genetic material to the next generation is performed with a fitness function. Recombination and mutation are simulated by exchange of genetic material and random modification. One of the benefits of a genetic algorithm is the ability to adapt to a changing environment [13].

This process can be applied to a population of images that evolve from one generation to the next. The key aspect in using genetic algorithms to create aesthetic images is to choose an appropriate fitness function. However, when choosing a fitness function one has to be careful to maintain enough creativity within the system [7]. For creating aesthetic imagery, aesthetic measures suggest themselves as a possible way of measuring fitness.

Coevolutionary algorithms maintain two or more populations that influence each other. The artist Steven Rooke used an algorithm that maintained populations of images and critics to produce images such as figure 2 [10].

The capability of such algorithms to produce sophisticated natural forms makes them an interesting candidate for research. The premise of using such systems to encode and represent complex data for the purpose of information visualization should be further explored [10]. However, due to their dynamic nature, genetic algorithms are not easy to work with. When researching into the topic, Greenfield was surprised to find that "very few of our ideas for articulating aesthetic criteria via fitness functions actually produced aesthetic imagery with this system" [7]. 


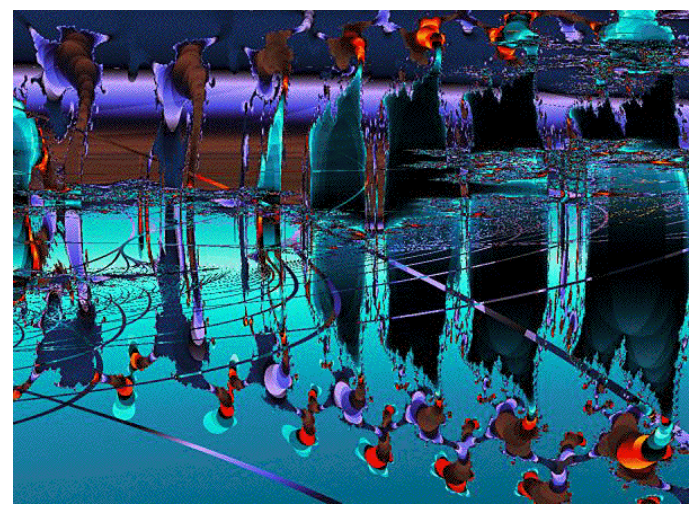

Figure 2: Artwork "Skaters" by Steven Rooke [10].

\section{Aesthetics and User Experience}

The value that aesthetics can have for information visualization is most apparent in its effect on the user. This section deals with approaches for the evaluation of aesthetic visualizations, observed results and their implications.

Tateosian et al. state that an effective visualization should attract and hold a viewers attention [21]. Since aesthetics generate attraction and capture the viewer's attention, positive effects on visualizations should be measurable.

Working on a model for the evaluation of visualizations Cawthon and Moere recognized that "there are complexities to understanding affect within the user experience of information visualization" [2]. The conventional metrics of participant task timing and the quantified fulfillment of goals do not seem to capture all the aspects of user experience. Instead of measuring how fast requested data can be retrieved, visualizations should be evaluated by the gain in experience and peripheral knowledge.

\section{"It is only through our emotions do we unravel problems, as the human emotional system is intertwined with our cognitive abilities." [16]}

This insight by Norman reflects the view that a user, who is attracted towards an object, will be more likely to think creatively in order to solve the problems presented by it [2]. Positive affect is likely to improve decision making and creativity, which is an important prerequisite for generating new insights [9].

It could be suggested that aesthetics in information visualization can facilitate a greater mental immersion into the underlying data. Studies in the area of virtual reality have shown that a higher level of immersion leads to better performance [17].

Research on the topics of aesthetics and usability has already lead to notable results in the field of human computer interaction. In a study conducted by Tractinsky et al. [22] students were presented with various ATM systems. The aesthetics of the systems were determined by different interface layouts. Their usability was influenced by several factors such as latency or irresponsive interface elements. The students were asked to rate the systems by appearance and ease of use. The gathered data showed a strong correlation between the perceived interface aesthetics and the perceived usability of the system.

Another study investigating the connection between aesthetics and usability was carried out by Cawthon and Moere [3]. An online survey was used to collect data about eleven different visualizations. All visualizations were representing the same hierarchical data. The participants were asked to rank the visualizations on a scale from "ugly" to "beautiful". Additionally, the users were given tasks in the form of questions about the underlying dataset. In order to accurately capture the effect of aesthetics, Cawthon and Moere introduced the metrics of task abandonment and erroneous response. These metrics were found to be correlated to aesthetic preference, suggesting that users approach aesthetic visualizations more thoroughly and with greater patience.

The preliminary results of experiments by Healey and Enns show varying aesthetic preferences. Covering a range of artistic styles, the study used impressionist, abstract and non-photorealistic images. The majority of viewers participating in this study preferred realistic images over abstract ones. Nonetheless, these viewers attributed artistic merit to the abstract images. A smaller number of viewers showed a preference for abstract imagery [8]. From the response to their ambient visualizations Skog et al. came to the conclusion that basing a visualization on an artistic style does not necessarily hinder - but might even support - the readability and comprehension of the visualization [19].

Information visualization is an interdisciplinary field and its users come from a variety of backgrounds [23]. This can make the design of visualizations very challenging. However, if visualization succeeds at creating aesthetic appeal, resulting in a more involved and patient user, it could be an important tool for bridging interdisciplinary gaps.

In order to realize the potential of aesthetics in information visualization a user-centered design approach needs to be adopted. Different user models have to be taken into account when evaluating aesthetic effect. The testing process needs to become a central part of the development of visualizations [2].

One of the central aspects of visualization is that it offers new perspectives on the underlying data. Fishwick notes that "this desire for alternate perspectives is consistent with art theory and practice" [5]. From this standpoint, research into aesthetic visualizations holds great value, 
since it explores new ways of presenting the underlying data to the user.

\section{Conclusions}

Recognizing that aesthetics is an unsolved problem of information visualization, this paper set out to explore existing approaches. At first, the problem was examined from an analytical perspective, focusing on the development of models to measure aesthetics. Thereupon, a selection of practical approaches for creating aesthetic visualizations was introduced. Finally, the paper identified positive effects of aesthetic visualizations on the user. This overview could be used as a starting point for further research.

Future work could include a more detailed look at aesthetic measures. The different measures should be tested with a common set of visualizations. This would give an opportunity to observe how the different measures behave on the same input. Ideally, this would lead to approximately equivalent results. However, it could be that some measures are better suited for assessing certain visualizations than others. The pattern measure proposed by Klinger and Salingaros might be better at capturing the subtle patterns in impressionist brush strokes. Then again, hereditary combinatorial entropy might turn out to be better when working with the compositions of geometric shapes inspired by abstract art. Evidently, this would pose a problem if aesthetic measures are to be used as intrinsic quality measures for visualizations.

In any case, it is likely that the presented measures can only offer a starting point in the search for an adequate aesthetic measure. Improved results might be achieved through the combination of several different approaches into one metric. It is also possible that a better aesthetic measure for information visualization can be found by taking the complexity of the underlying data into account rather than focusing solely on the generated image. Ultimately, a refined measure would have to be verified with a survey of a representative group of users.

\section{References}

[1] G. D. Birkhoff. Aesthetic Measure. Harvard University Press, 1933.

[2] N. Cawthon and A.V. Moere. A conceptual model for evaluating aesthetic effect within the user experience of information visualization. In $I V$ '06: Proceedings of the conference on Information Visualization, pages 374-382, Washington, DC, USA, 2006. IEEE Computer Society.

[3] N. Cawthon and A.V. Moere. The Effect of Aesthetic on the Usability of Data Visualization. In Proceedings of the 11th International Conference Information Visualization, pages
637-648. IEEE Computer Society Washington, DC, USA, 2007.

[4] C. Chen. Top 10 unsolved information visualization problems. IEEE Comput. Graph. Appl., 25(4):12-16, 2005.

[5] P. A. Fishwick. Exploring multiple visualization perspectives with aesthetic computing, September 2003.

[6] P. A. Fishwick, S. Diehl, J. Prophet, and J. Löwgren. Perspectives on aesthetic computing, April 2005.

[7] G.R. Greenfield. Computational aesthetics as a tool for creativity. In Proceedings of the 5th conference on Creativity \& cognition, pages 232-235. ACM New York, NY, USA, 2005.

[8] C.G. Healey and J.T. Enns. Perception and Painting: A Search for Effective, Engaging Visualizations. IEEE COMPUTER GRAPHICS AND APPLICATIONS, 22:10$15,2002$.

[9] A.M. Isen. The influence of positive and negative affect on cognitive organization: Some implications for development. Psychological and biological approaches to emotion, pages 75-94, 1990.

[10] G. Judelman. Aesthetics and inspiration for visualization design: bridging the gap between art and science. In Information Visualisation, 2004. IV 2004. Proceedings. Eighth International Conference on, pages 245-250, 2004.

[11] A. Klinger and N.A. Salingaros. A pattern measure. ENVIRONMENT AND PLANNING B, 27(4):537-548, 2000.

[12] H.G. Liddell, R. Scott, H.S. Jones, R. McKenzie, and E.A. Barber. A Greek-English lexicon. Clarendon Press Oxford, 1940.

[13] M. Mitchell. An Introduction to Genetic Algorithms. Bradford Books, 1996.

[14] J. Nešetřil. Aesthetics for Computers, or How to Measure Harmony. The Visual Mind II, 1:35-58, 2005.

[15] D.C.L. Ngo, A. Samsudin, and R. Abdullah. Aesthetic measures for assessing graphic screens. J. INF. SCI. ENG, 16(1):97-116, 2000.

[16] D.A. Norman. Emotional Design: Why We Love (or Hate) Everyday Things. Basic Books, 2004.

[17] D. Raja, D. Bowman, J. Lucas, and C. North. Exploring the Benefits of Immersion in Abstract Information Visualization. In Proc. Immersive Projection Technology Workshop, 2004.

[18] J. Rigau, M. Feixas, and M. Sbert. Informational aesthetics measures. IEEE Comput. Graph. Appl., 28(2):24-34, 2008.

[19] T. Skog, S. Ljungblad, and LE Holmquist. Between aesthetics and utility: designing ambient information visualizations. In Information Visualization, 2003. INFOVIS 2003. IEEE Symposium on, pages 233-240, 2003.

[20] T. Staudek and P. Machala. Recent exact aesthetics applications. In International Conference on Computer Graphics and Interactive Techniques, pages 241-241. ACM Press New York, NY, USA, 2002.

[21] L.G. Tateosian, C.G. Healey, and J.T. Enns. Engaging viewers through nonphotorealistic visualizations. In Proceedings of the 5th international symposium on Nonphotorealistic animation and rendering, pages 93-102. ACM Press New York, NY, USA, 2007.

[22] N. Tractinsky, AS Katz, and D. Ikar. What is beautiful is usable. Interacting with Computers, 13(2):127-145, 2000.

[23] J. J. van Wijk. Bridging the gaps. IEEE Comput. Graph. Appl., 26(6):6-9, 2006. 